\title{
Design and evaluation of a simulated wound management course for postgraduate year one surgery residents
}

\author{
Xin Qi ${ }^{\text {Corresp., Equal first author, } 1}$, Rui He ${ }^{\text {Equal first author, } 1}$, Bing Wen $^{1}$, Qiang $\mathbf{L i}^{1}$, Hongbin Wu $^{\text {Corresp. } 2}$ \\ 1 Plastic Surgery and Burns, Peking University First Hospital, Beijing, China \\ 2 Institute of Medical Education/National Centre for Health Professions Education Development, Peking University, Beijing, China \\ Corresponding Authors: Xin Qi, Hongbin Wu \\ Email address: 04983@pkufh.cn,wuhongbin@pku.edu.cn
}

Background: It is vital to cover wound management knowledge and operations in the early stages of resident training. With this in mind, a simulated wound management course for postgraduate year one surgery residents (PGY1s) was designed and its effectiveness was evaluated.

Methods: A retrospective quasi-experimental method was used. PGY1s in 2014 constituted the control group, and PGY1s in 2015 and 2016 constituted the intervention group. The course given to the control group comprised didactic teaching followed by deliberate practice plus immediate personalized feedback. The newly designed course given to the intervention group was reconstructed and disassembled into four components according to the simulation-based mastery learning model, which were baseline test, interactive learning, basic skills practice, and reflective learning. The same performance assessments were used in the control and intervention group, including process measurement and outcome measurement.

Results: The process measurement showed that the intervention group's scores were significantly higher in the "dissociation of subcutaneous tissue" and "quality of suturing and knots". The outcome measurement showed that the accuracy of debridement was greatly improved and both key and total suture numbers were significantly higher in the intervention group.

Conclusions: Simulation-based mastery learning was incorporated into our proposed course framework, promoting the learning outcome of PGY1s 


\section{Design and evaluation of a simulated wound 2 management course for postgraduate year one 3 surgery residents}

4 Xin Qi $M D^{\mathrm{a}^{*+}}$, Rui He $M D^{\mathrm{a}+}$, Bing Wen $M D^{\mathrm{a}}$, Qiang Li $M D^{\mathrm{a}}$ and Hongbin $\mathrm{Wu}$

$5 \quad P h D^{\mathrm{b}^{*}}$

6 a Department of Plastic Surgery and Burns, Peking University First Hospital, Beijing, China

7 bInstitute of Medical Education/National Centre for Health Professions Education Development,

8 Peking University, Beijing, China

$9+$ Rui He and Xin Qi contributed equally and are the co-first authors

$10 *$ Corresponding authors

11 Xin Qi, 04983@pkufh.cn, Department of Plastic Surgery and Burns, Peking University First

Hospital, 8 Xishku Street, Beijing, 100034, China ORCiDs:0000-0003-1124-2374

HongbinWu,wuhongbin@pku.edu.cn, Institute of Medical Education/National Centre for

Health Professions Education Development, Peking University, No. 38 XueYuan Road, Beijing, 100191, China 


\section{Abstract}

26 Background: It is vital to cover wound management knowledge and operations in the early

27 stages of resident training. With this in mind, a simulated wound management course for

28 postgraduate year one surgery residents (PGY1s) was designed and its effectiveness was

29 evaluated.

30 Methods: A retrospective quasi-experimental method was used. PGY1s in 2014 constituted the 31 control group, and PGY1s in 2015 and 2016 constituted the intervention group. The course given

32 to the control group comprised didactic teaching followed by deliberate practice plus immediate

33 personalized feedback. The newly designed course given to the intervention group was

34 reconstructed and disassembled into four components according to the simulation-based mastery

35 learning model, which were baseline test, interactive learning, basic skills practice, and reflective

36 learning. The same performance assessments were used in the control and intervention group,

37 including process measurement and outcome measurement.

38 Results: The process measurement showed that the intervention group's scores were

39 significantly higher in the "dissociation of subcutaneous tissue" and "quality of suturing and

40 knots". The outcome measurement showed that the accuracy of debridement was greatly

41 improved and both key and total suture numbers were significantly higher in the intervention 42 group.

43 Conclusions: Simulation-based mastery learning was incorporated into our proposed course

44 framework, promoting the learning outcome of PGY1s. It has the potential to be adapted for 45 other surgical training sites for residents in China. 


\section{Introduction}

Traditional surgical resident training has long followed the classical Halstedian teaching model of "see one, do one, teach one." However, significant changes have occurred in the training of surgical residents in China with the broadening of simulation-based training (Willis \& Van Sickle, 2015) and competency-based medical education (Li \& Wang, 2013). In the non-

54 55 67 errors. threatening, confidential, and "psychologically safe" (Fanning \& Gaba, 2007; Rudolph et al., 2008) training environment of simulation labs, trainees can continually improve their surgical skills with deliberate practice (Ericsson, 2008; McGaghie et al., 2011).

Wounds vary from minor vulnera (e.g., abrasions, lacerations) to severe life- or limbthreatening injuries. Wound management is a challenge faced by surgeons. If not carefully dealt with, clinical interventions could result in adverse effects on the skin, deep soft tissues, and even organs. Surgical site infections are the most typical wound, with an incidence of $0.4 \%-17.8 \%$ (World Health Organization, 2018). Currently, wound management training is distributed across different disciplines with different patterns. Trainees are mostly specialists or nurses, while medical students and residents, as major frontline staff, have insufficient opportunities to participate in training (Yim et al.,2014; Lupon et al., 2019).Wound management is an essential and required basic skill for postgraduate year 1 residents (PGY1s) in surgery training, and it is necessary to create a simulation-based wound management course for PGY1s to avoid medical

To effectively implement standardized training for surgical residents, Peking University First Hospital (PKUFH) established a surgery school in 2014, which was an important 
70 innovation in China (Qi et al., 2016). Plastic surgeons were assigned to instruct the simulation-

71 based wound management course. The course content included basic skills and the concept of

72 debridement and closure, which was delivered in the classic pattern of didactic teaching followed

73 by deliberate practice. However, the 2014 summative assessment did not yield satisfactory

74 results. We found that despite PGY1s had learned various basic technical skills during

75 undergraduate training in China, they were unable to effectively integrate these skills when

76 facing a complex simulated situation. Therefore, simple technical skills training was not suitable

77 for the residents, and specific training requirements were identified to allow the trainees to train

78 in a more clinically authentic environment, that is, decision-oriented procedural performance

79 regarding wound management.

80 In this study, we designed and dynamically revised a wound management course by

81 adapting simulation-based mastery learning (SBML) model aiming to improve the wound

82 management ability of PGY1s. The objective of this article is to describe the development,

83 implementation, evaluation and improvement of the course and investigate the effect of the

84 course by assessing PGY1s' learning outcomes.

85 Materials and Methods

86 Study Design and Participants

87 This was a retrospective quasi-experimental study. Participants of the wound management course

88 were PGY1s at PKUFH in 2014, 2015, and 2016, excluding those who did not complete the

89 course or the summative assessment. All PGY1s can enter the surgical training curriculum only

90 if they have passed the surgical residency entry examination, the criteria of which remained the

91 same for each year of the study. By the end of the course, learners were to be able to conduct

92 debridement and ultimately close the wound. Course contents included basic skills (asepsis and 
93 instrument identification, knot tying, suturing, excision, debridement, dissociation, wound

94 closure, skin flaps, etc.), and the knowledge of debridement and closure. The course contents had

95 been collectively evaluated and reviewed by experts in the surgery school to ensure their quality.

96 In the skills lab, a simulated procedural training was adopted using the pattern of small group

97 animal surgery. Instructors, examiners, learning objectives, and assessment criteria were

98 consistent across the three years. The simulation-based exercises were carried out in the same

99 skills lab, and the duration of the course was 240 minutes. The performance of two groups who

100 completed both assessments were used to evaluate the impacts of different simulation-based

101 courses. The overview of this study is described in Figure 1.

102 The two groups are as follows:

103 1. Control group: In 2014, the course for PGY1s was organized as a 60-minute didactic 104 teaching session including an operative demonstration, followed by 180 minutes of deliberate 105 practice plus immediate personalized feedback. A summary was given at the end. Rabbits under 106 general anesthesia were used for procedural training.

107 2. Intervention group: Based on the results in 2014, the course was reconstructed to include

108 four components of SBML: baseline test, interactive learning, basic skills practice, and reflective

109 learning. The process emphasized learner-directed small group learning with instructor

110 facilitation. A low-fidelity simulator was adopted and cadaveric pork belly skins were used for 111 procedural training.

112 The study was approved by the Institutional Review Board of PKUFH (2018-123). The 113 skills lab has the license issued by Beijing Municipal Commission of Science and Technology 114 for the use of experimental animals.

115 Simulation-based Mastery Learning Intervention 
116 According to the learning theory foundations of SBML (McGaghie \& Harris, 2018), the course

117 (for more details, see supplement 1) was designed following three learning theory foundations:

118 behavioral, constructivist, and social cognitive (Table 1).

119 Component 1. Baseline Test (10 Minutes)

120 PGY1s were required to perform a complete excision of the "dumbbell" necrotic tissue

121 within 10 minutes and close the wound (Fig. 2). Later, they were required to consider how to

122 repair a defect with the shape of two connected triangles. Starting from the classic case of plastic

123 surgery, clinical problems could stimulate their learning requirements and interests. Through the

124 baseline test, instructors could have a deeper understanding of their knowledge, skills, and

125 attitude so as to calibrate the learning direction and teaching strategies in the following parts.

126 Component 2. Interactive Learning (60 Minutes)

127 PGY1s were encouraged to review their performance on the blackboard and share their

128 thinking process and selection basis. Then instructors speculated about the PGY1s' mental

129 approaches through observation and prepared their plans accordingly. The concepts of

130 debridement and closure were communicated through narration and interrogation between

131 instructors and PGY1s. The role of the instructor in this component was a facilitator.

Component 3. Basic Skills Practice (80 Minutes)

PGY1s were expected to complete the closure of round, square, and triangular wounds. By approach (Nikendei et al., 2014), the instructor offered hands-on deliberate practice focusing on

practical issues. In this component, the instructor mainly corrected their basic skills (behaviors), discerned possible problems, and assessed their understanding of Component 2.

\section{Component 4. Reflective Learning (90 Minutes)}


The instructor provided feedback based on the completion of Component 3, expanded the

140 wound closure content, focusing on the principles and concepts of various local flaps, as well as

141 certain aesthetic issues (e.g., dog ear). Subsequently, PGY1s continued to complete round,

142 square, and triangular wound closures, or more complex and challenging wounds. They were

143 encouraged to communicate and collaborate with each other and offer help to others along with

144 the instructor. Deficiencies in cognition and procedure were exposed based on their

145 performances. The instructor observed the entire process and facilitated continuous reflection by

146 the PGY1s.

147 Performance Assessment

148 Our course is part of the entire surgical training curriculum, so the summative assessment

149 comprises one round of objective structured clinical examination (Fig. 3), which is held seven

150 months after the course. PGY1s' performances were assessed from the aspects of both the

151 process and the outcome. The examiners were three attending doctors from different

152 departments, who received training before the assessment. In detail, an instrument with a 10-

153 point rating scale modified from Objective Structured Assessment of Technical Skills (Hopmans

154 et al., 2014) was used to assess the PGY1s' surgical operation process according to six aspects

155 (maintaining a sterile field, knowledge and handling of instruments, quality of excision, quality

156 of debridement, dissociation of subcutaneous tissue, and quality of suturing and knots). Three

157 examiners graded the process of each resident, and average scores were obtained to serve as

158 overall scores for the process measurement. The outcome measurement was made using a

159 checklist focusing on five aspects (residual marking of incision margin, depth to deep fascia,

160 spindle resection, dog ear treatment, subcutaneous suture), along with the recording of total

161 suture number counts and key suture number counts. The same three examiners who graded the 
162 instrument of the process measurement also observed and discussed until consensus the marking

163 of all the areas of the outcome measurement instrument (five aspects and suture number counts).

164 Statistical Analysis

165 All results are expressed as mean and standard deviation (mean $\pm \mathrm{SD}$ ). Categorical variables

166 were analyzed with a Chi-square test, nonnormally distributed continuous variables were

167 analyzed with a Mann-Whitney test, normally distributed continuous variables were analyzed

168 with a one-way ANOVA, and pairwise comparison was performed using a least significant

169 difference test. The statistical analysis was performed using SPSS 22.0 software (IBM

170 Corporation, USA). A p-value of less than 0.05 was regarded as statistically significant.

171 Results

172 Participants

173 In 2014, 2015, and 2016, 31, 39, and 37 PGY1s were included, respectively, but only 23, 29, and

17428 completed the course (Table 2$)$. There were no significant differences in their ages $(\mathrm{p}=0.242$, $175 \mathrm{~F}=1.447)$.

176 Baseline Test

177 The wound closure performance of the PGY1s trained in 2015 was better than those trained 178 in 2016. Furthermore, spindle resection occurred significantly less frequently among the 2015 179 PGY1s than the 2016 PGY1s (Table 3).

180 Performance Assessment

$181 \quad$ Process Measurement

182 The results indicate the increased trend in scores over the three study years (for more details, 183 see supplement 2). The scores of the 2015 and 2016 PGY1s were significantly higher than those 184 of the 2014 PGY1s for the quality of debridement and dissociation of subcutaneous tissue. None 
185 of the 2014 PGY1s performed the dissociation of subcutaneous tissue, so they scored zero for

186 this dimension. Based on the assessment results of 2015, dissociation of subcutaneous tissue

187 training was reinforced for the 2016 PGY1s, and they got a higher score than 2015 PGY1s (Fig.

188 4).

189 Outcome Measurement

190 Compared with those in 2014, the PGY1s in 2015 and 2016 showed no obvious

191 improvement in the residual marking of incision margins (Table 4). However, their debridement

192 accuracy (no spindle resection and more depth of deep fascia) did improve greatly. Both key and

193 total suture numbers were significantly higher. Nobody in 2014 or 2015 dealt with dog ear

194 treatment, but some PGY1s in 2016 did. However, under the premise of an increased choice of

195 excision along the remarking and dissociation of subcutaneous tissue, the PGY1s in 2015 and

1962016 aimed to close the wound as soon as possible and did not choose a subcutaneous suture.

\section{Discussion}

198 Mastery learning is a rigorous approach to competency-based education that requires students to 199 engage in educational activities that have clear learning objectives and through deliberate 200 practice to reach the minimal passing standard before advancing to another unit. SBML is in 201 perfect alignment with such an educational philosophy; it significantly improve clinical skills for 202 all participants and leads to skills retention (Motola et al., 2013). Although simulation-based 203 education was adopted before 2014 in the PKUFH, the concept of mastery learning was 204 introduced in 2015 and 2016, and the effect has significantly improved. In the meantime, the 205 learning process has become self-paced, proactive, and self-reflecting (Bandura A, 1997). 206 Emergency departments take in many skin and soft tissue (SST) injuries (Health Protection 207 Agency, 2005; Nawar et al., 2007; Jones et al., 2012). An estimated 82.8\% of traumatic injuries 
208 are accompanied by soft-tissue injuries, complicating potential limb salvage in patients

209 (Madubueze et al., 2011). SST is both the starting and ending point of almost all surgeries. From

210 a technical skill and equipment perspective, soft-tissue coverage procedures can be performed

211 effectively even in low-resource settings (Wu et al., 2016). Therefore, as novices, it is necessary

212 for PGY1s to receive early training for complex, rare, and critical SST wounds in skills labs in

213 order to identify key issues and initiate proper preliminary treatment.

214 Today, surgical training places most weight on technical skills training, simulation, and

215 learning by doing. The course in our study comprises mainly basic surgical skills except for local

216 flap, but simple technical training cannot meet the clinical requirements of PGY1s. Since 2015,

217 we have implanted questions into typical clinical cases in the baseline test to facilitate active

218 study for PGY1s. After the baseline test, their perception, interpretation, and construction of

219 meaning were motivated by authentic problems (McGaghie \& Harris, 2018). Naturally, the

220 learning model changed from didactic teaching to situated interactive learning, and the

221 instructor's role changed to facilitator. Through interactive learning, PGY1s can benefit from the

222 development of their cognitive processes and improve the efficiency and effectiveness of their

223 behavioral skill acquisition by observing the practice of others (Ericsson \& Pool, 2016). Decision

224 making was introduced into interactive learning and gradually applied in basic skills practice and

225 reflective learning for further training of clinical reasoning. The procedure was deconstructed

226 into deliberate practice with basic surgical skills and experiential learning of repair and

227 reconstruction concepts, so that the degree of difficulty gradually increased. Specific,

228 informative feedback increased PGY1s' skills performance in a controlled setting (Issenberg et

229 al., 2005). Through grasping and transforming experiences, PGY1s' competence improved.

230 Therefore, this new course has the basic elements of SBML (McGaghie et al., 2010), which are 
231 the baseline test (with units sequentially ordered by increasing difficulty), engagement in

232 educational activities, measurement of whether outcomes meet or exceed the mastery standard, 233 and deliberate practice.

234 In SBML, deconstruction means to decompose the sophisticated skill into simple tasks to 235 lower the cognitive load. Interactive learning focuses on the concept of debridement and closure 236 through experiential learning, intending to establish PGY1s' clinical reasoning about wound 237 management. Basic skills practice focuses on surgical skills training. Reflective learning is for 238 reconstruction, manifested as concrete experience-reflection-active experimentation. Through the 239 process of deconstruction and reconstruction, the overall cognitive load will decrease with 240 practice, as some components of the skill begin to become automatic, which will transform into 241 competence (Motola et al., 2013).

242 Furthermore, although wound tension reduction was included in the course content, it was 243 submerged in an overwhelming amount of information in 2014, causing no PGY1s to attempt 244 dissociation of subcutaneous tissue. Since 2015, however, deconstruction of the original course 245 content helped strengthen this aspect. The necessity of subcutaneous suture decreased as a direct 246 consequence of the reduced wound tension, which benefited from excision along the remarking 247 and dissociation of subcutaneous tissue, and therefore, most 2015 and 2016 PGY1s omitted this 248 step as it was no longer necessary. PGY1s in 2015 and 2016 achieved significant improvement in 249 dissociation of subcutaneous tissue.

250 Instructors can adjust the content, difficulty, and complexity of the simulation-based 251 intervention in real time according to the baseline test. Although the hospital's resident selection 252 criteria remain constant every year, residents may perform differently even in basic skills 253 depending on their learning experience and educational background (Barsuk et al, 2012). This 
254 phenomenon was revealed in the baseline test results, which showed that the 2016 PGY1s' basic

255 understanding of wound management was relatively inadequate compared with that of the 2015

256 PGY1s. In light of this information, the instructors were required to provide opportunities for

257 individualized learning by dynamically adjusting the course content. An individual's level of

258 prior knowledge may affect their learning and teaching methods, so PGY1s with high levels may

259 afford and require more self-regulation and reflection, whereas PGY1s with low levels would

260 benefit from more guidance (Chernikova et al., 2020). In 2015, more time was allotted for

261 training in repair and reconstruction skills, whereas in 2016 the predetermined course content

262 was completed, leaving more time to review basic concepts. We also revised the relevant course

263 content based on the results of the previous year to check for deficiencies and fill in the gaps in

264 each iteration. Debridement and subcutaneous dissociation were emphasized in 2016, and

265 process measurement was changed accordingly. Meanwhile, with the continuous improvement of

266 the course content, both key and total suture numbers were significantly higher in 2015 and 2016 ,

267 indicating that both the surgical proficiency and the holistic view of wound closure had been

268 obviously improved; some PGY1s in 2016 even managed the dog ear. Therefore, although there

269 were some differences in the baseline test between PGY1s in 2015 and 2016, their performance

270 assessment was approximately equal.

271 This study adopted a low-technology task training simulation. The effectiveness of the

272 simulation depends on the demands of the clinical task. It is important that simulations can

273 capture or represent a variety of patient problems and conditions (Issenberg et al, 2005).

274 Simulations for novice trainees may not require simulators with high mechanical fidelity or

275 simulations that are overly complex (Motola et al, 2013). For PGY1s to master the basics of flap

276 design and implementation, porcine skin maybe the most cost-effective and efficient choice 
277 (Hassan, Hogg \& Graham, 2014). The materials are relatively cheap and easy to obtain. Such 278 simulations (using pork belly skin, for example), which are low in authenticity, can achieve high 279 quality outcomes, possessing the potential to be transferred to courses in other surgical training 280 programs in China.

281 Limitations

282 First, the study was conducted in a surgical training site for residents with a small number of 283 participants. Second, as the baseline test was not included in 2014, the actual baseline of the 284 control group before the intervention was unknown. We were more concerned about the 285 consistency of outcome standards than entry standards. Third, the Mastery Education in 286 Medicine (Cohen et al., 2015) model was not referred to at the beginning of the process of 287 curriculum design. As our new course is still in the exploratory stage, future studies will aim to 288 be designed strictly according to mastery learning standards by setting minimum passing scores 289 and allowing continued repeated practice or study of an educational unit until reaching mastery, 290 and post-intervention impacts of the course should be further improved and evaluated with the 291 outcomes measured in the Kirkpatrick model levels 3 and 4. Fourth, the summative assessment 292 was conducted 7 months after the intervention; although PGY1s had few opportunities to 293 manage clinical cases of complex wounds during this period, there still could be certain effects 294 on the ability of wound management for PGY1s. Finally, PGY1s were assessed in a skills lab, 295 not in an actual clinical situation. Future studies may attempt to develop this as a trustable 296 professional activity and conduct the assessments in the authentic clinical settings.

\section{Conclusions}


299 Wound management is relatively stable and requires further improvement Our proposed course

300 framework, which incorporated SBML, could promote the wound management competence of

301 PGY1s. Given its effectiveness and feasibility, it has the potential to be adapted in other surgical

302 training sites for residents in China.

\section{Acknowledgements}

304 The authors acknowledge the PGY1s and staffs at PKUFH for their cooperation and support.

\section{Definitions}

306 Dumbbell: Flat shape with big ends and small middle, similar to dumbbell.

307 Local flap: Consists of skin and subcutaneous tissue that is harvested from a site nearby a given

308 defect while maintaining its intrinsic blood supply.

309 Deep fascia: A layer of dense connective tissue that can surround individual muscles and groups

310 of muscles to separate into fascial compartments.

311 Dog ear: A one-sided mound of redundant tissue, which is seen after the repair of certain skin

312 lesions and defects.

313 Debridement: Removal of dead, damaged, or infected tissue to improve the healing potential of

314 the remaining healthy tissue.

315 Spindle resection: Surgical removal of the target tissue using an incision with the shape of a 316 slender round rod with narrowed ends.

\section{References}


318 Bandura A. 1997. Self-efficacy: the exercise of control. New York: W.H. Freeman and 319 Company.

320 Barsuk JH, Cohen ER, Caprio T, McGaghie WC, Simuni T, Wayne DB. 2012. Simulation-based 321 education with mastery learning improves residents' lumbar puncture skills. Neurology.79(2): $322 \quad 132-137$.

323 Chandra DB, Savoldelli GL, Joo HS, Weiss ID, Naik VN. 2008. Fiberoptic oral intubation: the 324 effect of model fidelity on training for transfer to patient care. Anesthesiology.109(6):1007-1013.

325 Chernikova O, Heitzmann N, Stadler M, Holzberger D, Fischer F. 2020. Simulation-based 326 learning in higher education: A meta-analysis. Review of Educational Research. 90(4):499-541.

327 Cohen ER, McGaghie WC, Wayne DB, Lineberry M, Yudkowsky R, Barsuk JH. 2015.

328 Recommendations for Reporting Mastery Education Research in Medicine (ReMERM). 329 Academic Medicine.90(11):1509-1514.

330 Ericsson K, Pool R. 2016. Peak: Secrets from the new science of expertise. Boston: Houghton 331 Mifflin Harcourt.

332 Ericsson KA. 2008. Deliberate practice and acquisition of expert performance: a general 333 overview. Acadmic Emergency Medicine. 15: 988-994.

334 Fanning RM, Gaba DM. 2007. The role of debriefing in simulation-based learning. Simulation in 335 Healthcare. 2(2):115-125.

336 Grober ED, Hamstra SJ, Wanzel KR, Reznick RK, Matsumoto ED, Sidhu RS, Jarvi KA.

337 2004.The educational impact of bench model fidelity on the acquisition of technical skill: the use 338 of clinically relevant outcome measures. Annals of Surgery.240(2):374-381.

339 Hamstra SJ, Brydges R, Hatala R, Zendejas B, Cook DA. 2014. Reconsidering fidelity in 340 simulation-based training. Academic Medicine.89(3):387-392. 
341 Hassan Z, Hogg F, Graham K. 2014. A 3-dimensional model for teaching local flaps using

342 porcine skin. Annals of Plastic Surgery.73(4):362-363.

343 Health Protection Agency. 2005. Health Protection in the 21st century - understanding the

344 burden of disease: preparing for the future. Part 10: injuries. Health Protection Agency.

345 Hopmans CJ, den Hoed PT, van der laan L, van der Harst E, van der Elst M, Mannaerts GH,

346 Dawson I, Timman R, Wijnhoven BP, IJzermans JN. 2014. Assessment of surgery residents'

347 operative skills in the operating theater using a modified Objective Structured Assessment of

348 Technical Skills (OSATS): a prospective multicenter study. Surgery. 156: 1078-1088.

349 Issenberg SB, McGaghie WC, Petrusa ER, Lee Gordon D, Scalese RJ. 2005. Features and uses

350 of high-fidelity medical simulations that lead to effective learning: a BEME systematic review.

351 Medical Teacher. 27(1):10-28.

352 Jones AP, Barnard AR, Allison K, Wright H. 2012. Review of emergency department wound

353 management in soft tissue trauma - is there a plan?. Journal of Wound Care.21(9):431-438.

354 Li HC, Wang C. 2013. Reform direction of medical education in China: implementing

355 “competency-based” medical education. Chinese Medical Journal (English). 126: 3203-3204.

356 Lupon E, Turrian U, Malloizel-Delaunay J, Bura-Rivière A, Grolleau JL. 2019. Internes en

357 médecine et cicatrisation des plaies: une étude descriptive multicentrique entre février et avril

3582018 [Medical residents and wound healing: A French national survey]. Journal of Medecine

359 Vasculaire. 44(5):324-330.

360 Madubueze CC, Chukwu CO, Omoke NI, Oyakhilome OP, Ozo C. 2011. Road traffic injuries as

361 seen in a Nigerian teaching hospital. International Orthopaedics.35(5):743-746.

362 McGaghie WC, Harris IB. 2018. Learning theory foundations of simulation-based mastery

363 learning. Simulation in Healthcare. 13(3S Suppl 1):S15-S20. 
364 McGaghie WC, Issenberg SB, Cohen ER, Barsuk JH, Wayne DB. 2011. Does simulation-based

365 medical education with deliberate practice yield better results than traditional clinical education?

366 A meta-analytic comparative review of the evidence. Academic Medicine. 86: 706-711.

367 McGaghie WC, Issenberg SB, Petrusa ER, Scalese RJ. 2010. A critical review of simulation-

368 based medical education research: 2003-2009. Medical Education.44(1):50-63.

369 Motola I, Devine LA, Chung HS, Sullivan JE, Issenberg SB. 2013. Simulation in healthcare

370 education: a best evidence practical guide. AMEE Guide No. 82. Medical Teacher.

$371 \quad 35(10): \mathrm{e} 1511-\mathrm{e} 1530$.

372 Nawar, E.W., Niska, R.W., Xu, J. 2007. National Hospital Ambulatory Medical Care Survey:

3732005 emergency department summary. Advances in Data. 386:1-32.

374 Nikendei C, Huber J, Stiepak J, Huhn D, Lauter J, Herzog W, Jünger J, Krautter M. 2014.

375 Modification of Peyton's four-step approach for small group teaching - a descriptive study. BMC

376 Medical Education. 14:68.

377 Qi X, Li Y, Li HC, Wang Y, Liu YC. 2016. A preliminary study on the role of surgery school the

378 standardized training of surgical residents. Chinese Journal of Medical Education. 36: 764-767.

379 Rudolph JW, Simon R, Raemer DB, Eppich WJ. 2008. Debriefing as formative assessment:

380 closing performance gaps in medical education. Academic Emergency Medicine. 15(11):1010-

3811016.

382 Willis RE, Van Sickle KR. 2015. Current status of simulation-based training in graduate medical 383 education. Surgical Clincs of North America. 95: 767-779.

384 World Health Organization. 2018. Global guidelines for the prevention of surgical site infection, 385 second edition. Available at 
386 https://apps.who.int/iris/bitstream/handle/10665/277399/9789241550475-eng.pdf?ua=1

387 (accessed December 2020)

388 Wu HH, Patel KR, Caldwell AM, Coughlin RR, Hansen SL, Carey JN. 2016. Surgical

389 Management and Reconstruction Training (SMART) course for international orthopedic

390 surgeons. Annals of Global Health.82(4):652-658.

391 Yim E, Sinha V, Diaz SI, Kirsner RS, Salgado CJ. 2014. Wound healing in US medical school

392 curricula. Wound Repair and Regeneration. 22(4):467-472. 
Figure 1

The study overview

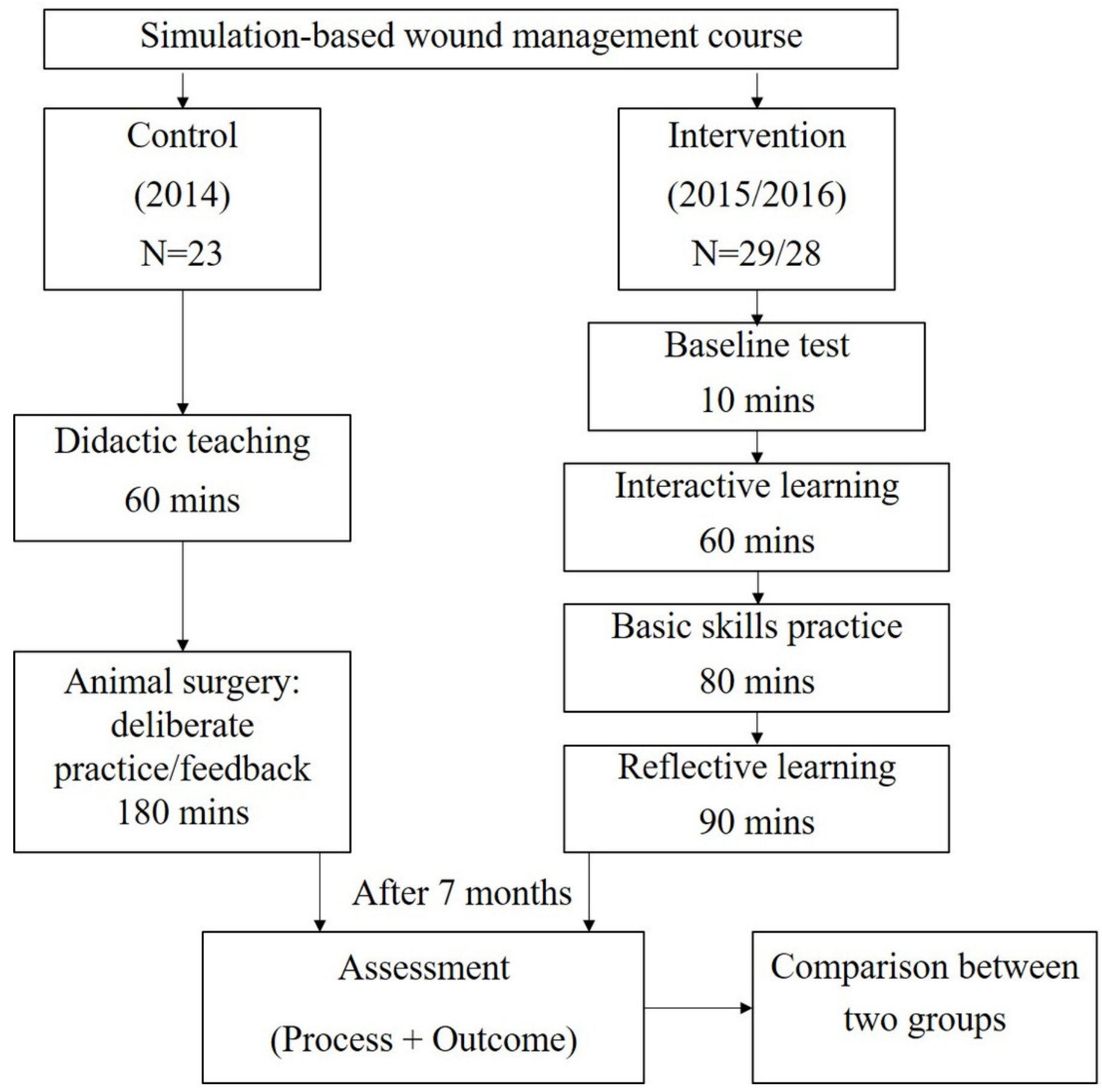




\section{Figure 2}

\section{Baseline test}

(A)The necrotic area was marked. (B) The PGY1s were required to perform a complete excision of the lesion and a primary closure of the wound. They were expected to leave flaps $a$ and $b$ open and suture the two key points-point $c$ to point $a$ and point $b$ to point $d$. (C) The expected results. (D) Typical error: spindle excision (normal tissue was not retained and incision tension was increased). (E) Typical error: no preoperative planning (cut mechanically along the edge, and only sutured the low tension areas)
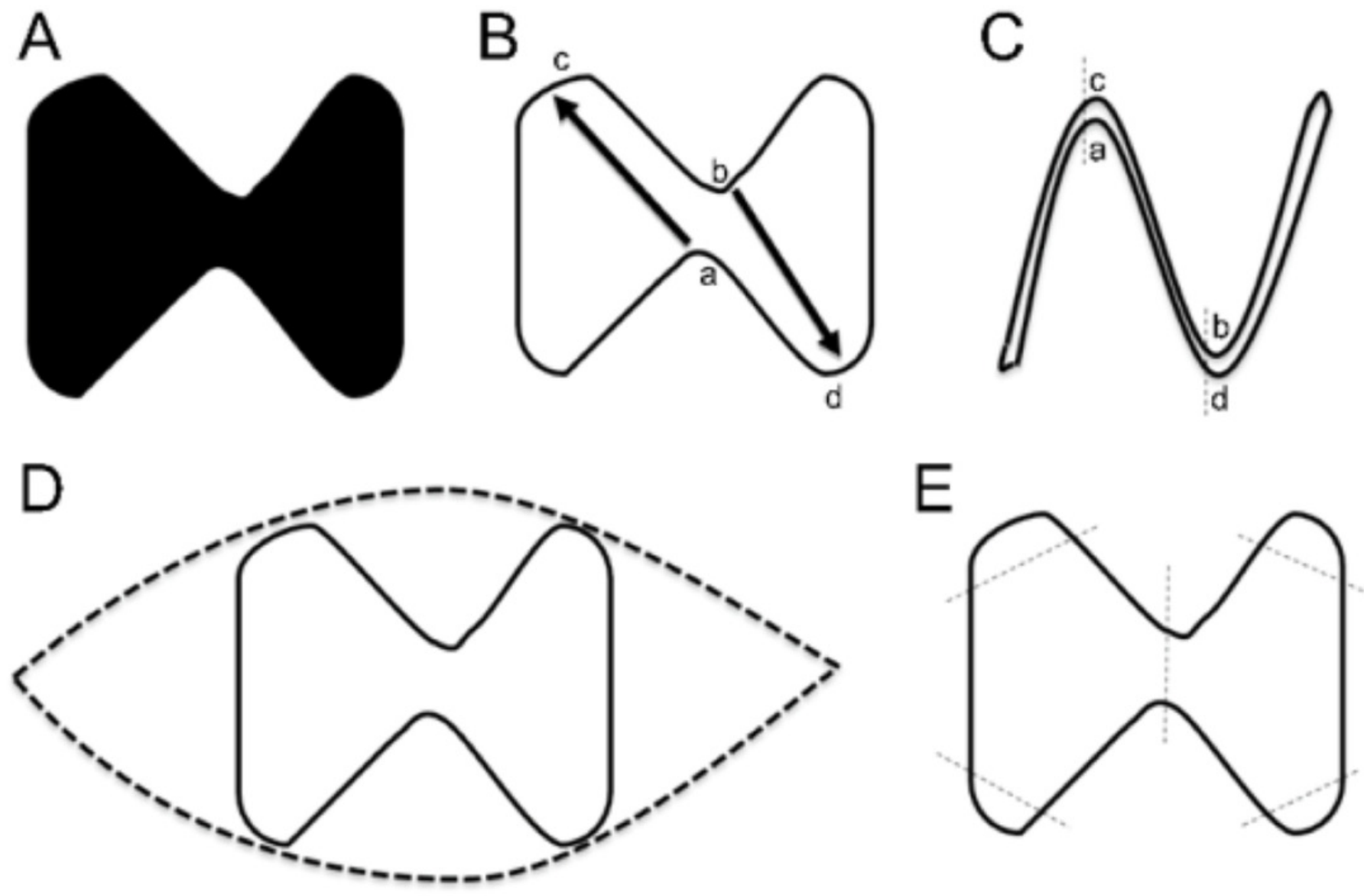


\section{Figure 3}

\section{Outcome measurement}

(A) An irregular area of $10 \times 3.8 \mathrm{~cm}^{2}$, marked on cadaveric pork belly skin, was regarded as the necrotic part, affecting the deep fascia. The PGY1s were required to perform a primary suture after debridement within 15 min. (B) The PGY1 excised along the margin, but residual marking was visible, and wound bed wasn't deep enough to the fascia (C) The box showed 4 key sutures, and the cycle marked the dog ear. 


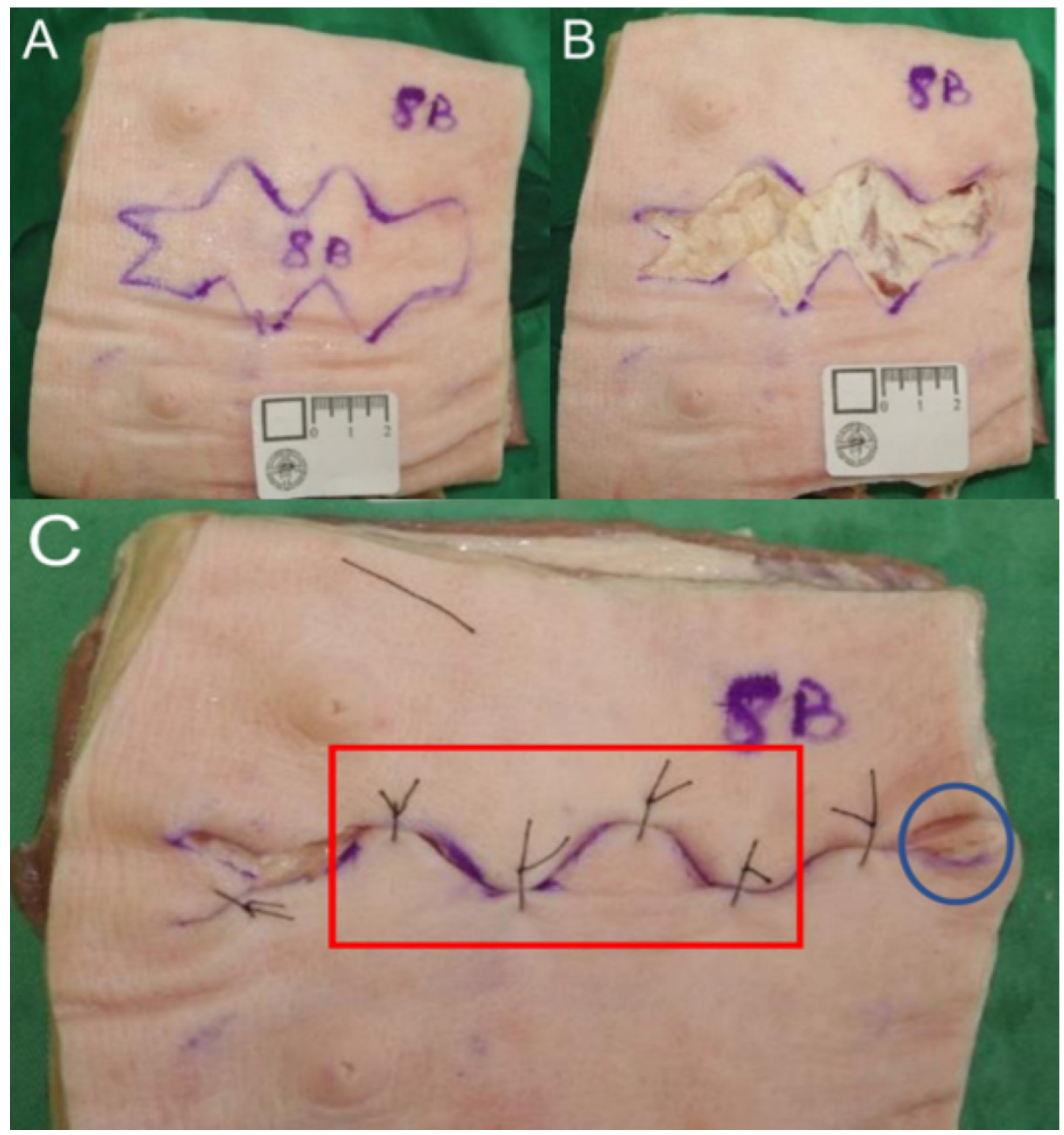


Figure 4

Process measure

$p$ values were calculated by the least significant difference tests

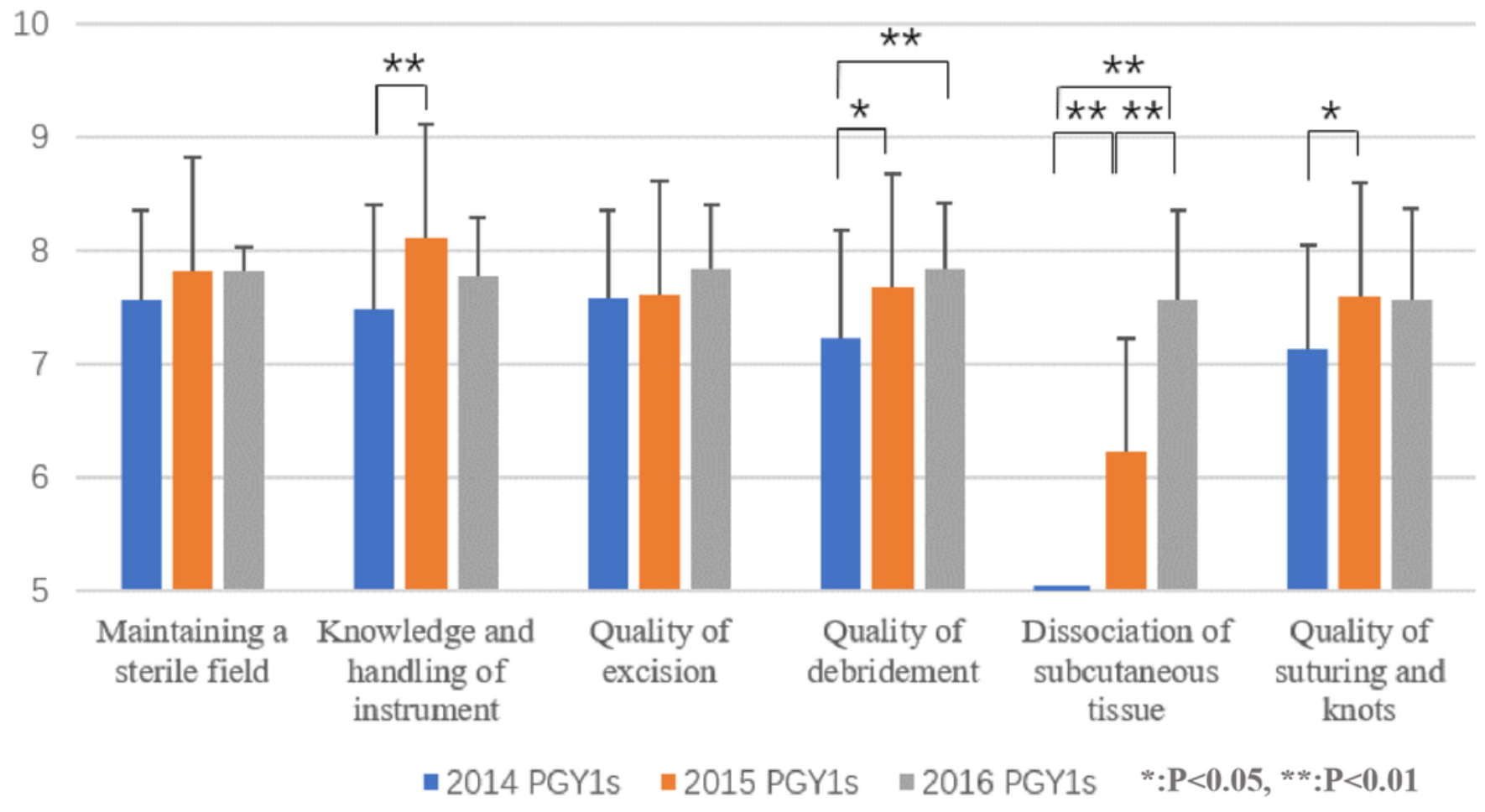




\section{Table 1 (on next page)}

Learning theory foundations of the course 


\begin{tabular}{cl} 
Theory Foundations & Wound Management Skills Acquired and Assessed Using SBML \\
\hline Behavioral & Asepsis and instrument identification, knot tying, suturing, excision, \\
debridement, dissociation, wound closure, skin flaps, etc. & Recognize clinical signs, critical thinking, problem solving: identify a \\
Constructivist & lesion's range and depth, develop a treatment plan, be aware of protecting \\
& skin and soft tissue, reduce wound tension, use local flap technique to \\
& repair the wound, etc. \\
Secial Cognitive & Rective learning, peer education (resident as instructor), increasing \\
clinical self-efficacy about complicated wounds, acquiring & communication and collaboration skills.
\end{tabular}




\section{Table 2 (on next page)}

Demographic information of the postgraduate year 1 surgery residents trained from 2014 to 2016

Note: *: $p$ value was calculated by the Chi-square tests, ${ }^{\Delta}: p$ value was calculated by one-way ANOVA. 
1

\begin{tabular}{cccccc}
\hline & Control group & & \multicolumn{2}{c}{ Intervention group } & $p$-value \\
\cline { 2 - 2 } \cline { 5 - 6 } & 2014 & & 2015 & 2016 & \\
\cline { 5 - 6 } Gender:no.(\%)male & $23 / 23(100)$ & & $29 / 29(100)$ & $26 / 28(92.9)$ & $0.200^{*}$ \\
Age:mean $\pm \mathrm{SD}($ year) & $24.78 \pm 1.70$ & & $24.55 \pm 1.21$ & $25.25 \pm 1.78$ & $0.272^{\Delta}$ \\
\hline
\end{tabular}

2 


\section{Table 3(on next page)}

Baseline test results of the postgraduate year 1 surgery residents trained in 2015 and 2016

Note: $C=$ local flap transfer; $\mathrm{D}=$ spindle resection; $\mathrm{E}=$ necrosis resection, aimless and convenient suture; $p$ values were calculated by the Chi-square tests. 


\begin{tabular}{cccc}
\hline Dimensions & $2015(\mathrm{n}=34)$ & $2016(\mathrm{n}=30)$ & $p$-value \\
\hline $\mathrm{C}[\%(\mathrm{n})]$ & $47.0(16)$ & $10.0(3)$ & 0.001 \\
$\mathrm{D}[\%(\mathrm{n})]$ & $11.8(4)$ & $43.3(13)$ & 0.004 \\
$\mathrm{E}[\%(\mathrm{n})]$ & $41.2(14)$ & $46.7(14)$ & 0.659 \\
Wound closure [\% (n)] & $11.8(4)$ & $0(0)$ & 0.116 \\
\hline
\end{tabular}

1

2 


\section{Table 4 (on next page)}

Outcome measurement

Note: Residual $=$ residual marking of incision margin; Depth $=$ depth to deep fascia; Spindle $=$ spindle resection; Dog ear $=$ dog ear treatment; Subcutaneous = subcutaneous suture; Numbers $=$ Suture numbers. $*: p$ values were calculated by the Chi-square tests, ${ }^{\star}: p$ values were calculated by the Mann-Whitney tests. 
1

\begin{tabular}{|c|c|c|c|c|c|c|c|}
\hline \multirow{2}{*}{\multicolumn{2}{|c|}{ Dimensions }} & \multirow[b]{2}{*}{2014} & \multirow[b]{2}{*}{2015} & \multirow[b]{2}{*}{2016} & \multicolumn{3}{|c|}{$p$-value } \\
\hline & & & & & $\begin{array}{c}2014 \text { vs. } \\
2015\end{array}$ & $\begin{array}{c}2014 \text { vs. } \\
2016\end{array}$ & $\begin{array}{c}2015 \text { vs. } \\
2016\end{array}$ \\
\hline \multicolumn{2}{|c|}{ Residual [\% (n)] } & $30.4(7)$ & $34.5(10)$ & $28.6(8)$ & $0.757^{*}$ & $0.884^{*}$ & $0.631 *$ \\
\hline \multicolumn{2}{|c|}{ Depth $[\%(n)]$} & $30.4(7)$ & $82.8(24)$ & $71.4(20)$ & $0.000^{*}$ & $0.004 *$ & $0.308^{*}$ \\
\hline \multicolumn{2}{|c|}{ Spindle $[\%(n)]$} & $30.4(7)$ & $0.0(0)$ & $0.0(0)$ & $0.002 *$ & $0.002 *$ & l \\
\hline \multicolumn{2}{|c|}{ Dog ear $[\%(n)]$} & $0.0(0)$ & $0.0(0)$ & $14.3(4)$ & l & $0.117^{*}$ & $0.052^{*}$ \\
\hline \multicolumn{2}{|c|}{ Subcutaneous $[\%(n)]$} & $56.5(13)$ & $10.3(3)$ & $14.3(4)$ & $0.000^{*}$ & $0.001 *$ & $0.706^{*}$ \\
\hline \multirow{2}{*}{$\begin{array}{c}\text { Numbers } \\
{[\mathrm{n}(\min , \max )]}\end{array}$} & Key numbers & $0(0,4)$ & $3(0,4)$ & $3(0,4)$ & $0.000^{\Delta}$ & $0.014^{\Delta}$ & $0.702^{\Delta}$ \\
\hline & Total numbers & $3(0,12)$ & $5(0,14)$ & $5(0,12)$ & $0.019^{\Delta}$ & $0.022^{\Delta}$ & $0.917^{\Delta}$ \\
\hline
\end{tabular}

2 